\title{
LA ONTOLOGÍA DE ROMAN INGARDEN ACERCA DE LOS OBJETOS TEMPORALES: ANÁLISIS Y PROYECCIONES
}

\author{
Mario Cuneo Bosco \\ Instituto de Filosofía, Universidad de Los Andes \\ mcuneo@miuandes.cl
}

\begin{abstract}
Resumen / Abstract
El análisis del tiempo es un tema de primera importancia en la investigación filosófica. Roman Ingarden, quien fue un destacado discípulo de Husserl, desarrolló una interesante teoría acerca de los objetos temporalmente determinados. Se presentan en este artículo los elementos fundamentales de esta teoría, como las dificultades que implica el intento de Ingarden de definir el tiempo con independencia de la subjetividad. Finalmente, se argumenta que es posible aplicar parte de los conceptos desarrollados por Ingarden para avanzar en el análisis del tiempo, en tanto éste surge en la propia subjetividad, donde se equilibran la permanencia y la transitoriedad.
\end{abstract}

Palabras Clave: Ingarden, tiempo, procesos, objetos que permanecen en el tiempo, continuos, sucedentes.

\section{ROMAN INGARDEN'S ONTOLOGY OF TEMPORAL OBJECTS: ANALYSIS AND PROJECTIONS}

The analysis of the time is a matter of first importance in the philosophical investigation. Roman Ingarden, who was a distinguished disciple of Husserl, developed an interesting theory about temporally determined objects. In this paper are presented the fundamental elements of his theory and the difficulties implied in the Ingarden's intent to define time with independence of the subjectivity. Finally, it is argued that it is possible applying partially the concepts developed by Ingarden in order to progress in the analysis of the time, as it emerges in the own subjectivity, where exist an equilibrium of endurance and transience.

KEY WORDS: Ingarden, time, process, objects enduring in time, continuants, occurrents.

\section{Introducción}

$\widehat{R A}$ Roman Ingarden desarrolló un profundo análisis del tiempo, como parte de sus investigaciones acerca de la controversia entre "idealistas" y "realistas" sobre la existencia del mundo. En su investigación, Ingarden desarrolla una "ontología existencial", con el fin de establecer los posibles modos de ser. En una primera instancia, Ingarden desarrolla su análisis sin hacer mención explícita del tiempo, llegando a determinar un primer conjunto de modos posibles de ser. Pero una vez alcanzado este resultado, Ingarden considera necesario examinar la temporalidad, porque, al menos 
desde el punto de vista de la experiencia prefilosófica, parece pertenecer al modo de ser más íntimo de los objetos conectados con el tiempo, que aparecen en el dominio del "mundo real".

En este trabajo se presentan los elementos fundamentales del análisis ingardiano de los objetos temporales, a saber, procesos, eventos y objetos que perduran en el tiempo, incluyendo sus argumentos para incluir a los seres vivos en este último tipo de objeto. Junto con ello, se van indicando una serie de dificultades que resultan si se toman estos objetos en los términos absolutos que propone Ingarden. Como punto principal, cabe mencionar que procesos y objetos que perduran, más que conceptos separados son complementarios, particularmente en el caso de los seres vivos, donde permanencia y cambio parecen estar naturalmente entrelazados en un equilibrio inestable. Por ello, a diferencia de Ingarden, y volviendo un tanto al punto de vista de Husserl, propongo realizar un análisis de la temporalidad desde la circunstancia de la propia subjetividad, pero tomando en cuenta los conceptos de Ingarden de procesos y objetos que perduran al modo de límites. En esta línea, se argumenta que es posible avanzar en el análisis del tiempo, en tanto surge en la propia subjetividad, donde se da una multiplicidad de elementos que constituyen un entrelazamiento de duraciones, y no un agregado de instantes.

\section{Husserl e Ingarden}

En su estudio, Ingarden considera explícitamente a los empiristas ingleses, a Kant, y principalmente a Husserl, de quien fue discípulo en Göttingen. Respecto de este último, es indispensable describir, aunque sea mínimamente, la discrepancia que separa a Ingarden de Husserl, porque ésta constituye un elemento fundamental de toda su investigación, incluyendo su análisis del tiempo. Para Ingarden, el idealismo trascendental de Husserl es uno de los intentos más significativos de resolver la controversia acerca de la existencia del mundo real, pero indica expresamente que "[...] me parece muy improbable que la solución propuesta por Husserl sea correcta”(Ingarden 1964, p. 9)².

Ingarden presenta su discrepancia con Husserl en las primeras secciones del libro Time and Modes of Being, al examinar sus conceptos de conciencia "pura" y de "mundo real", atendiendo fundamentalmente a lo publicado en el libro Ideas relativas a una Fenomenología Pura (Ingarden 1964, pp. 9-16). Si bien un análisis de este último texto excede ampliamente el alcance de este trabajo, cabe mencionar algunos pasajes donde

$1 \quad$ Un análisis más detallado de la posición de Ingarden respecto de Husserl se encuentra en el capítulo 2 de Mitscherling, Roman Ingarden's Ontology and Aesthetics, (1997). Cabe mencionar también el capítulo II de la parte I del libro Beyond Ingarden's Idealism/Realism Controversy with Husserl - The New Contextual Phase of Phenomenology (Tymieniecka, 1976).

2 Todas las citas de Ingarden las he traducido al español a partir de la versión en inglés. 
Husserl afirma claramente la primacía de la conciencia pura como realidad fundamental, dado que esta tiene la capacidad de "poner entre paréntesis" el mundo natural entero. Esta investigación deja siempre en duda al mundo real, como por ejemplo en el $\S 46$, que lleva el muy significativo título de "Indubitabilidad de la percepción inmanente, Dubitabilidad de la trascendente" (Husserl 1993, pp. 104-107). A modo de brevísimo sumario, podemos citar el siguiente párrafo del § 76:

La reducción fenomenológica nos dio por resultado el reino de la conciencia trascendental como reino de un ser "absoluto" en un sentido muy preciso. Es este ser la categoría radical del ser en general (o, en nuestro lenguaje, la región radical) en que tienen sus raíces todas las demás regiones del ser, a que se refieren por su esencia, de la que por tanto dependen esencialmente todas (Husserl 1993, p. 169) ${ }^{3}$.

A este respecto, Ingarden indica que la conciencia pura se da en la percepción inmanente, lo que garantiza su carácter de indudable, y en cambio el mundo real no goza de esta condición, pues los objetos que lo constituyen son por esencia no-inmanentes (Ingarden 1964, pp. 15-16). Por consiguiente, si se adopta como punto de partida este flujo de conciencia o experiencia consciente "pura", para así tratar de alcanzar por vía indirecta a los componentes del mundo real, de acuerdo a Ingarden se termina por llegar siempre a una incompatibilidad metodológica. Cualquier intento en esta dirección estaría condenado a fracasar, porque la experiencia consciente "pura" está fuera de toda duda posible (en la línea de Descartes) y en cambio el mundo real está siempre sujeto a esta.

Frente a esta dificultad, Ingarden considera que, en lugar de investigar directamente el estatus del mundo real frente a la conciencia pura, es necesario delimitar con mayor precisión estos dos reinos del ser, y determinar las posibles relaciones de dependencia o independencia entre ambos, considerándolos como dos modos de ser igualmente posibles. Tal como lo señala Wladyslaw Strózewski, antes de analizar si existe un "mundo real", Ingarden realiza un estudio ontológico que no se dirige a los hechos, sino a las posibilidades puras (Strózewski 2010, p. 14).

\section{El tiempo concreto}

Ingarden comienza su análisis de la temporalidad indicando que al hablar de "tiempo" se refiere al tiempo concreto, el que distingue del tiempo abstracto definido por símbolos matemáticos (como sería el caso del símbolo " $\mathrm{t}$ " de las fórmulas de la física), y del tiempo ordinario, que resulta de la comparación de varios tiempos concretos. Ingarden indica que el tiempo concreto es un tiempo lleno, que contrasta con el tiempo vacío de los símbolos. El tiempo concreto es "llenado" por lo que sucede, por lo que tiene

He incorporado el subrayado a la cita original, para destacar la primacía de la conciencia pura. 
efecto o lo que perdura "en él". Este tiempo es absoluto, en el sentido de que este tiempo es del objeto en sí mismo, el cual existe en el objeto, y no es solo subjetivamente condicionado (Ingarden 1964, p. 100). Así, este tiempo concreto es propio y esencial de los objetos temporales, se vincula ontológicamente con su modo de ser, y por tanto puede ser analizado directamente.

Cabe aquí comparar, aunque sea de manera muy resumida, este "tiempo concreto", con el concepto del tiempo en Kant, autor que es mencionado por el propio Ingarden en varios pasajes de Time and Modes of Being. Según la doctrina de Kant, tiempo y espacio no son entes, sino "formas de nuestra sensibilidad", condiciones a las que se tiene que someter los objetos que captamos por los sentidos (Torretti 2005, p. 91). El tiempo según Kant no es un concepto extraído de la experiencia, sino su base "a priori”, aquella representación necesaria que sirve de base a todas las intuiciones(Kant 1998, p. 74: A 31 / B 46). Kant menciona explícitamente la necesidad del tiempo como base para poder representarse que algo existe simultáneamente o sucesivamente. De este modo podríamos entender el tiempo como la referencia fundamental para que sea posible la experiencia sensible "ordenada". Este tiempo "a priori" es intuido, es decir, representado de forma inmediata en la mente, pero siempre asociado al ejercicio de sus capacidades sensibles (Torretti 2005, pp. 230-232). Así, este tiempo no es derivado, como un común denominador de todo lo sensible, sino que es primero, porque de este nace la posibilidad de conocer a los objetos sensibles, aunque accedemos a su realidad en cada percepción sensible. El tiempo-espacio sería la forma de procurarnos acceso a objetos, en la medida en que permiten una primera ordenación de los datos sensibles.

Ingarden, en cambio, plantea que es posible acceder directamente a un"tiempo concreto", tal como se da en los objetos temporalmente determinados, dejando de lado el problema de las "formas de la sensibilidad", las que considera ajenas a la esencia de cada objeto. Por ello Ingarden señala que no considera importante distinguir entre tiempo "experimentado" y "no-experimentado", sino atender primordialmente al objeto en sí-mismo (Ingarden 1964, p. 101).

La noción de "tiempo concreto" también implica una diferencia fundamental con Husserl, ya que Ingarden plantea que es algo posible de investigar con independencia de la conciencia "pura". Husserl claramente plantea lo contrario, por ejemplo, en las Lecciones, en su $§ 1$ indica que:

Lo que nosotros admitimos no es [...] la existencia de un tiempo del mundo, la existencia de una duración de las cosas, etc., sino el tiempo que aparece, la duración que aparece como tal. Estos son los datos absolutos, dudar de los cuales sería absurdo. Ciertamente que con ello asumimos también un tiempo que existe pero que no es el tiempo del mundo de la experiencia, sino del tiempo inmanente del curso de la conciencia (Husserl 2002, p. 26) ${ }^{4}$.

$4 \quad$ Roman Ingarden hace referencia a varios textos de Husserl, como por ejemplo en sus “Consideraciones Preliminares"(Ingarden 1964, p. 9 n. 3), pero no menciona las Lecciones. 
No obstante, hay que hacer notar un punto de relativa coincidencia entre ambos autores, puesto que Ingarden tampoco se dirige directamente al "mundo real", sino que analiza los objetos temporales posibles, en cuanto a su estructura existencial implicada en su idea. La pregunta ingardeana acerca del tiempo podría exponerse como sigue: ¿Cuáles deberían ser las características esenciales de los objetos temporales, con independencia de si realmente existen o no? La cuestión respecto de la realidad efectiva de dichos objetos es materia de un análisis posterior, pero que, para Ingarden, exige el conocimiento previo de estas características.

\section{Los objetos temporalmente determinados 5}

Ingarden distingue los siguientes objetos temporalmente determinados, que se diferencian en cuanto a sus modos de ser: los objetos que perduran en el tiempo, los procesos y los eventos (Ingarden 1964, p. 101).

Aunque Ingarden inicia su análisis por los eventos, me parece más claro exponer primero su descripción de los procesos, porque de acuerdo al mismo Ingarden éstos revelan la esencia del tiempo de una mejor manera que el modo de ser de los eventos (Ingarden 1964, p. 109).

Ingarden da algunos ejemplos de procesos, como la carrera de un atleta en una competición, el desarrollo de un organismo, la vida de una persona, etc. Cada proceso comprende "un conjunto continuo de fases que incesantemente se expanden mientras el proceso está en operación" y el objeto que "con el paso del tiempo, está siendo constituido en estas fases como el sujeto de las propiedades que constituyen el proceso". Estos son dos "lados" de una misma entidad (Ingarden 1964, p. 107). La propiedad general de un proceso, en cuanto objeto, es ser un agregado temporalmente extenso de fases. Estas fases son continuamente transitorias, y se caracterizan por los siguientes momentos:

A. Una y solo una fase es siempre actual.

B. Una nueva fase tras otra siempre llega a ser actual.

C. Una fase actual continuamente va perdiendo su actualidad y una nueva fase, que está justo comenzando, va ganando actualidad.

No obstante, es importante considerar este texto al momento de comparar los análisis de ambos autores.

5 En este punto se hace una descripción que coincide en algunos aspectos con la sección "Ingarden on enduring objects and processes" del artículo de Marek Piwowarczyk (2010). No obstante, el enfoque es diferente, por cuanto dicho artículo se centra en la controversia respecto del substancialismo, y en mi artículo la investigación se centra en el análisis de los objetos temporales, en especial de los seres vivos como objetos que permanecen. 
D. En el instante en que la fase actual ocurre, las fases antecedentes están ya extintas, pero tuvieron existencia, mientras que las fases subsiguientes todavía no existen, pero están viniendo a la existencia (a ser actuales).

E. En el instante en que la última fase alcanza actualidad, el proceso ha pasado. Pero no todo proceso tiene una fase final ${ }^{6}$.

Ingarden agrega: "La totalidad de las fases de un proceso trascienden cada presente y la fase contenida en ese presente, y abarca un cierto segmento del pasado y también del futuro -naturalmente mientras el proceso está todavía desplegándose" (Ingarden 1964, p. 109).

De acuerdo con Ingarden, el despliegue de las fases de un proceso permite entender la esencia del tiempo, que describe como "transitoriedad", a saber, “...la perpetua transformación de la actualidad de algo presente en un enigmático "noseguir-estar-siendo-presente-nunca-más", mientras, al mismo tiempo, subsiste en algún modo de ser como algo pasado" (Ingarden 1964, p. 111). La necesaria continuidad que caracteriza a los procesos, en su progresiva agregación de fases sin cortes, solo es posible si entendemos al tiempo en esta perpetua transformación, y no como un agregado de puntos instantáneos, donde en términos estrictos solo existe lo que está contenido en el presente, no existiendo en absoluto ni pasado ni futuro. Bajo dicho esquema, como se verá al tratar de los eventos, los procesos deberían ser un agregado de elementos discretos perfectamente separados, donde siempre hay solo un punto en el tiempo, el cual es ahora. Pero esto va en contra de la propia esencia de los procesos, que al ser continuos, parecen más bien corresponder a un tiempo extendido, donde algo pasado, aunque no es estrictamente actual, mantiene un modo de existencia, y donde algo futuro es más que una nada absoluta (Ingarden 1964, pp. 109-111).

Pero en este continuo, ¿cómo se diferencia lo que es presente, de lo que es pasado y futuro? Ingarden indica que "lo actual se caracteriza por ser directamente activo, o, expresado de otra forma, por la acción (la cualidad de producir un efecto)" (Ingarden 1964, p. 112) ${ }^{7}$. Agrega que esta posibilidad de la acción, más allá de la forma concreta que adopte, atraviesa todo lo que es actual, y es por tanto un momento primario que caracteriza la existencia presente. Me parece, sin embargo, que si atendemos al tiempo concreto tal como lo entiende Ingarden, es decir, al tiempo del proceso en sí mismo, resulta difícil distinguir intrínsecamente cada fase actual respecto de las otras, sin hacer referencia a algo externo al propio proceso, puesto que la acción es siempre acción sobre algo (otro). Una alternativa es separar dentro del mismo proceso dos o

6 Si bien no se realiza una traducción literal, se sigue en forma muy cercana el texto original, incluyendo las palabras en cursivas, tal como esta descripción se encuentra en Ingarden (1964, pp. 108-109).

7 He optado por traducir "operancy" utilizando la descripción más extensa indicada por el traductor al inglés del texto en Ingarden (1964, pp. 112 n. 21), por ser más ilustrativa que la palabra "operatividad" que en español tiene un sentido más bien tecnológico y funcional. 
más partes interrelacionadas, pero a su vez en cada parte habría que distinguir subpartes y así sucesivamente, cayendo en una división al infinito.En mi opinión, cabría señalar que desde el punto de vista del proceso, lo esencial es el desenvolvimiento de las fases en sucesión, y la actualidad de una fase determinada solo tiene lugar cuando el proceso entra en relación con algo otro. En consecuencia, el análisis de lo actual (y del significado de qué entendemos por "ahora") debería hacerse siempre atendiendo a la relación entre varios procesos separados, siendo preferentemente uno de éstos la propia corriente de conciencia que conoce de un determinado proceso, como se plantea al final de este artículo.

No obstante estas objeciones, hay que destacar la fina y rica descripción que hace Ingarden de los procesos, con su sucesión de fases que van progresivamente alcanzando y dejando la actualidad presente. Al respecto, me parece pertinente plantear que una investigación en la línea de lo señalado en las letras $\mathrm{C}$ y D de la descripción de los procesos debería permitir acceder a una mejor determinación de la naturaleza del tiempo y del fundamento de la duración.

Ingarden define los eventos como "la ocurrencia de un determinado estado de cosas o de una situación objetiva" (Ingarden 1964, p. 102). Menciona algunos ejemplos, como la llegada de un tren a la estación, el encenderse de una lámpara, la muerte de un hombre, etc. Los eventos no tienen duración, ocurren en un solo instante. No deben confundirse con procesos muy cortos o muy compactos en cuanto al desarrollo de sus fases, que permanecen durante algún tiempo. Hay también que separar conceptualmente el hecho de que algo ocurrió y su posible preparación, que corresponde a un proceso que sí tiene duración. Los eventos se relacionan con el tiempo limitado al "ahora", con su momento de actualidad (Ingarden 1964, p. 105), en contraste con los procesos donde se revela su momento de duración, tal como se indicó en el punto anterior. Por ello los eventos solo pueden mantenerse presentes como algo que existió por medio de lo que permanece más allá de ellos, y que conserva los efectos de su ocurrencia. Aunque Ingarden reconoce la dificultad de concebir como temporal (sujeto al tiempo) a algo que no tiene duración, sostiene que no es cuestionable que existen eventos instantáneos (Ingarden 1964, pp. 106-107).

Para entender esta existencia instantánea de los eventos, y atendiendo a los ejemplos que da Ingarden, se podría decir que un evento ha ocurrido cuando se ha dado algo que marca un antes y un después, pero de tal forma que el evento mismo no lo encontramos ni en el antes ni en el después. Así, podríamos considerar los eventos al modo de un límite, minimizando su "tiempo" hasta justo el borde donde ya no habría tiempo alguno. Todo lo ocurrido en este "ahora" limitado sería un evento. No obstante, esta idea debe ser entendida de manera meramente ilustrativa, porque corresponde más a una formulación sofisticada de un proceso muy compacto (que siempre conserva alguna duración), que al concepto de evento instantáneo (sin duración) planteado por Ingarden.

Considerando las dificultades antes señaladas, más que proponer una definición precisa de cómo los eventos se podrían dar en la existencia real, hay que contrastar su modo de ser con el de los procesos. Esta diferencia entre eventos y procesos se presenta muy claramente cuando Ingarden muestra la imposibilidad de considerar un proceso 
como un agregado de eventos. Para ello da el ejemplo de una serie de lámparas que se van encendiendo sucesivamente, dando la apariencia de un movimiento continuo, cuando en verdad se tiene una secuencia de hechos discretos (Ingarden 1964, pp. 110111). Esta ilusión es solo posible porque parte de lo que va ocurriendo (discretamente) es retenido por el observador, quien lo integra de este modo en una continuidad. Es el observador en este caso quien le da el carácter de continuo a algo que no lo es de suyo $^{8}$. Para Ingarden no hay tránsito posible entre eventos y procesos, porque ambos expresan dos concepciones diferentes de la experiencia del tiempo, a saber, el de la instantaneidad y el de la duración.

Ingarden comienza su análisis de los objetos que perduran en el tiempo mencionando como ejemplos una piedra, una casa, una montaña. También incluye a los seres vivos y a las personas, y adelanta que éstos son los objetos que nos permiten comprender la radical diferencia entre eventos, procesos y objetos que perduran, a pesar de las dificultades teóricas que surgen al analizar su particular modo de ser (Ingarden 1964, pp. 124-125). Se volverá sobre este tema en los puntos 5 y 6.

Los objetos que perduran se diferencian de los eventos en el hecho básico de que pueden durar más allá de los instantes en que los eventos están “encapsulados". En este aspecto, los objetos que perduran se asemejan a los procesos, pero se diferencian de ellos en que, en cada instante, se mantienen siendo los mismos, conservando su identidad, en contraste con la continua sucesión de fases de los procesos ${ }^{9}$.

Ingarden lleva adelante una detallada comparación entre procesos y objetos que perduran en el tiempo. Desde el punto de vista existencial, plantea que los objetos

8 Respecto de este ejemplo, se puede objetar que el encenderse de una lámpara no es estrictamente un evento, por cuanto si bien en la observación directa no podemos establecer una duración, ello sí lo podemos hacer por medio de instrumentos de medición más precisos que nuestra visión. Si nos ceñimos rigurosamente a la condición de que los eventos no tienen duración en absoluto, entonces no podremos encontrar en la naturaleza ningún fenómeno que podamos identificar como un evento. Desde esta perspectiva, los eventos serían posibles solo como una definición, por ejemplo, de que la guerra ha terminado. En el caso particular de la muerte de una persona, comprobamos que la persona ya ha muerto, pero el instante preciso probablemente nunca lo podremos determinar.

$9 \quad$ M. Piwowarczyk, siguiendo la comparación entre procesos y objetos que permanecen que hace Ingarden (1964, pp. 128-130), indica que se podría distinguir a los procesos de los objetos que perduran considerando que los primeros no son completamente presentes en cada momento de su existencia y en cambio los segundos sí son completamente presentes en cada momento de su existir (Piwowarczyk 2010, p. 163). No obstante, este esquema no es totalmente aplicable si consideramos a un objeto que permanece como una estructura formada por un núcleo esencial más sus estados que cambian en el tiempo, como indica Ingarden (1964, pp. 134-139). Adicionalmente, esta comparación nos lleva al problema de determinar qué es ser presente, es decir, a quién y cómo, porque implícitamente ser presente es ser presente a algo o a alguien. Este es uno de los temas que intento revisar en la parte final de mi artículo. Respecto de la interpretación de ser "totalmente presente" existen distintas posiciones, como se puede ver en Ingthorsson (2009). 
que perduran tienen preeminencia respecto de los procesos, porque estos no podrían existir sin un objeto que permanezca. Ingarden señala que los objetos que perduran son los "soportes" necesarios de los procesos ${ }^{10}$. En cambio, los objetos que perduran no requieren de los procesos para mantener su identidad (Ingarden 1964, pp. 126-128) ${ }^{11}$.

No obstante, los objetos que perduran no pueden estar completamente separados de los procesos. Porque, si bien desde el punto de vista de su relación con los procesos, los objetos que perduran se distinguen porque en principio pueden persistir perfectamente invariantes y sobrevivir a la mutabilidad del tiempo, esta persistencia no es al modo de los objetos puramente ideales, que están fuera del tiempo, y no en el tiempo (Ingarden 1964, pp. 128-129). Esta necesaria participación en las transformaciones temporales lleva a Ingarden a admitir que en principio son posibles varios tipos de objetos que perduran, desde los absolutamente inmutables a los que pueden cambiar en diversos grados (Ingarden 1964, p. 132). Por consiguiente, dado que, como se mencionó en el punto 4, es el despliegue de las fases de un proceso lo que permite entender la esencia de la temporalidad, los objetos que perduran en el tiempo no pueden estar completamente desconectados de los procesos. Y junto con los procesos, tenemos la posibilidad del cambio, que Ingarden entiende tanto como la modificación de propiedades del objeto, cuanto como el cambio que implica el paso desde la actualidad del presente a la noactualidad del pasado. Para explicar la posibilidad del cambio en un objeto que persiste, Ingarden indica que existen un núcleo invariante y una serie de estados sujetos al cambio. Los estados se definen como un conjunto de propiedades asociadas al objeto, que persisten por un determinado periodo de tiempo, pero que cambian mientras el objeto se mantiene siendo siempre el mismo (Ingarden 1964, pp. 133-134, 137). Las fronteras entre el núcleo invariante y los estados no son fijas, aunque hay un límite más allá del cual la existencia del objeto es afectada, hasta llegar a su destrucción (Ingarden 1964, p. 139). Me parece pertinente mencionar en este punto la cercanía de esta solución con el esquema de sustancia-accidente aristotélico, el que es mencionado en varias secciones de Time and Modes of Being ${ }^{12}$.

10 Traduzco "bearer" por soporte, ya que se trata de una clasificación existencial, y no metafísica, donde cabría utilizar más bien el término de agente.

11 Aunque se trata de un análisis existencial, debo hacer notar que, tal como el mismo Ingarden lo discute en algunos párrafos de estas páginas, hay una fuerte influencia de la física de partículas en esta posición, según la cual los procesos son resultado de la interacción entre partículas relativamente invariantes. Contra esto, hay que considerar las ecuaciones de onda de la mecánica cuántica.

12 Respecto de la influencia de Aristóteles en Husserl e Ingarden, se puede consultar: Mitscherling, Aristotelian Metaphysics and the Distinction between Consciousness and the Real World in Husserl and Ingarden (2010). De acuerdo con M. Piwowarczyk, los objetos que perduran son concebidos por Ingarden como "substancias clásicamente concebidas" (Piwowarczyk 2010, p. 163). 


\section{Los seres vivos como objetos que perduran}

El análisis antes descrito lleva a Ingarden a preguntarse si los seres vivos son un objeto que perdura o un conjunto complejo de procesos. En primera instancia, los vivientes se caracterizan por las múltiples transformaciones que presentan a lo largo de su desarrollo, donde es casi imposible dar un caso concreto de un elemento inmutable. Así, parecería no haber diferencia con el modo de ser de los procesos. No obstante, también hay que atender a las regularidades características que encontramos en cada especie biológica, que se repiten constantemente, y más notablemente, a los modos de comportamiento de las personas. Ingarden indica:

Es este rasgo de una determinada personalidad, el mismo a lo largo de una vida, y el modo siempre uno y el mismo una y otra vez, como el individuo dado resuelve la gran variedad de problemas en su vida (a menudo en situaciones opuestas), manifestándose y prevaleciendo sobre todos los obstáculos y catástrofes. Esta es la mejor evidencia de que un individuo viviente, y la persona humana en particular, es algo más y diferente de la suma de los eventos y procesos que lo han afectado (Ingarden 1964, p. 143).

Este "algo" más, que tiene la capacidad de diferir de lo que sería la tendencia del curso general del proceso, para Ingarden revela la existencia de una base esencial capaz de interactuar con su medio y de persistir a los cambios que lo afectan. Por ello describe a los individuos como un tipo de estructura estratificada cuyas capas "superiores" (o externas) son condicionadas tanto por su núcleo básico, como por las circunstancias de la vida (Ingarden 1964, p. 143). De esta forma, los seres vivientes tienen una identidad dinámica, en contraposición con una identidad estática (perfectamente inmutable). Esta identidad dinámica se caracteriza porque en ella los cambios solo afectan el modo y grado de desarrollo de los aspectos existenciales y fenoménicos de un cierto momento objetivo. En particular en el hombre, tenemos el desarrollo y la manifestación de una determinada cualidad o complejo de cualidades, pero que, de acuerdo con Ingarden, es de absolutamente lo mismo que estaba contenido embrionariamente al comienzo de todo el proceso (Ingarden 1964, pp. 145-146).

De esta forma, un individuo viviente se caracteriza por una especial combinación de persistencia y transitoriedad, cuyo completo sistema existencial es un equilibrio inestable entre su base perdurable (su núcleo esencial) y el estrato superficial de propiedades que se constituyen solo en el curso de su vida (sujetas a la transición y a la acción del medio). Este equilibrio está sujeto a fluctuaciones, y siempre puede llegar el momento donde este se altere al grado de llevar a la extinción del individuo. Esto para Ingarden revela un esencial defecto en el ser de los vivientes, y por tanto constituye un "momento", es decir, una propiedad específica de su modo de existencia (Ingarden 1964, p. 149).

Ingarden señala que los seres vivos, en cuanto objetos que perduran, trascienden la actualidad del puro presente, a pesar de que su existencia está siempre confinada al presente único. Ingarden indica que "la actualidad de su existencia es siempre como 
una estrecha fisura" (Ingarden 1964, pp. 151-152). En el caso especial de los individuos conscientes, esta fisura puede ser superada a través de los actos de recolección, retención, protención y anticipación ${ }^{13}$, los que van más allá del presente inmediato. Con estos actos, los individuos pueden trascender el presente estricto, abarcando un horizonte mayor que incluye, por una parte, el pasado propiamente tal, más lo que está justo pasando, y por otra, lo justo por suceder y lo que esperamos más allá en el futuro que prevemos. Sin embargo, debo hacer notar que para Ingarden la existencia de los vivientes está siempre confinada a un presente único que no se puede sobrepasar, por lo que esta trascendencia no significa que su modo de ser se asimile a la extensión temporal propia de los procesos. Ingarden precisa al respecto que los individuos conscientes pueden "penetrar el movimiento del tiempo y la síntesis de los cambios temporales", produciendo un cierto tipo de proyección sobre el incesante flujo del tiempo, que abre la posibilidad de generar un sentido unitario del individuo, solo accesible para los sujetos conscientes (Ingarden 1964, p. 155).

\section{Actualidad del análisis de los objetos temporales de Ingarden}

El aporte de Ingarden respecto de los objetos temporales, aunque no ha tenido la misma difusión que sus estudios en estética, ha sido considerado en algunos trabajos más recientes. Un ejemplo importante lo encontramos en el artículo "Continuants and Ocurrents" (Simons 2000a), que es particularmente citado acerca de este tema, y que presenta varias conexiones con la visión de Ingarden ${ }^{14}$.

Simons señala que un constante ${ }^{15}$ es cualquier objeto que existe en el tiempo y no tiene partes temporales (Simons 2000a, p. 59). Por otra parte, un sucedente es un objeto en el tiempo que tiene partes temporales, es decir, fases de duración que

13 Los términos de recolección, retención, protención y anticipación son usados por Husserl, y aunque Ingarden no hace aquí referencia a este autor, es muy probable que los tome de éste. Tomando en cuenta la nota del traductor en Ingarden (1964, p. 155 n. 51), retención se refiere al pasado inmediato consciente, "todavía vivo" que ha sido "hace un momento" (Husserl 1993, p. 179), en cambio la recolección es el recuerdo activo. Protención se refiere al futuro inmediato, también en el límite del horizonte del presente, pero ahora dirigido hacia adelante (Husserl 1993, p. 173), que a su vez se diferencia de la anticipación que es activa.

14 Peter Simons, entre otras materias, es un estudioso de la filosofía de Europa Central, y en particular de Austria y Polonia en los siglos XIX y XX. Adicionalmente, es de notar que el propio Simons cita el texto de Ingarden Time and Modes of Being en el artículo que se comenta.

15 He optado por traducir continuants por constantes en lugar de la traducción literal continuos, para acentuar su carácter de permanencia. En el caso de Occurrents he utilizado el neologismo sucedentes, que me parece más acertado que el literal ocurrentes o el término sucesos que no transmite adecuadamente la idea de cambio y movimiento. 
corresponden a los intervalos en los cuales éste perdura (Simons 2000a, p. 60). Es así evidente la cercanía de estos conceptos con los de objetos que perduran y procesos de Ingarden. En cuanto a los eventos, Simons los considera junto con los procesos, y por tanto son también sucedentes.

Pero aunque ambos autores reconocen la interrelación existente entre estos dos tipos de objetos, hay una diferencia radical entre Simons e Ingarden. Mientras para este último los objetos que perduran tienen la primacía, para Simons es un hecho que los sucedentes son las entidades fundamentales (Simons 2000a, pp. 60, 75). Dada esta primacía, una opción es considerar que realmente los constantes no existen, pero Simons indica que hasta ahora no ha sido posible mostrar cómo un lenguaje podría funcionar tratando a todos los particulares como sucedentes, por lo que considera necesario mantener la posición del sentido común de que los constantes existen (Simons 2000a, p. 62). Pero si los constantes existen, lo hacen en el tiempo, y como son los sucedentes los que por naturaleza tienen partes temporales, y en cambio los constantes no, el gran problema es determinar cómo los constantes pueden existir en el tiempo (Simons 2000a, p. 66). La propuesta de Simons es que los constantes existen en el tiempo porque existe al menos un sucedente que los "involucra" (Simons 2000a, p. 69), por lo que un constante tiene una existencia derivada. Simons propone que un constante es un "invariante", definido por el hecho de que los sucedentes que le son vitales (a dicho constante) se repiten en la forma de una relación de equivalencia en una serie de situaciones sucesivas. Por consiguiente, en el caso específico de los seres vivos, un constante es una abstracción que resume las equivalencias de los sucedentes que caracterizan su existencia, que se repiten en diferentes tiempos (Simons 2000a, pp. 72-73). Esta conclusión sería extensible a todo ente del mundo real, como una roca o una estrella, ya que la física, al menos hasta ahora, siempre ha encontrado una estructura interna de múltiples elementos en interacción, que al conservar su equilibrio, mantienen la identidad del conjunto.

Considerando lo anterior, los constantes se muestran muy cercanos a la idea de los seres vivos como objetos que perduran, establecida por Ingarden, cuando los caracteriza por un comportamiento típico que se repite una y otra vez en su interacción con el entorno, tal como se señaló en el punto 5. Pero hay aquí también una clara discrepancia, ya que Ingarden postula que este comportamiento es manifestación de un núcleo invariante esencial, en permanente equilibrio inestable con el medio, lo que se contrapone a la postura de Simons, que considera que lo realmente primero son los sucedentes, siendo los constantes una abstracción ${ }^{16}$.

Ahora bien, Simons describe a los seres vivos como constantes, considerando preferentemente el funcionamiento interno del organismo, como la respiración, el latir del corazón, etc. (Simons 2000a, p. 70). Esto no es casual, porque si los sucedentes

16 Si bien aclara que no es una abstracción en sentido matemático, sino una abstracción "atada" a sus ocurrentes vitales que existen en el tiempo y en el espacio (Simons, "Continuants and Occurrents", Part I, 2000a, p. 74). 
son el fundamento de los constantes, entonces estos no son sino la externalización reconocible de la invariancia de un fragmento de los sucedentes. Por consiguiente, para identificar a un constante nos debemos atener a lo que sucede dentro de los límites de un candidato a ser considerado dentro de este tipo de objetos. Pero esto podría no funcionar tan bien como parece, puesto que surge la pregunta acerca de cómo podemos, en principio, llegar a saber de dichas equivalencias. El mismo Simons señala en otro artículo que el hecho de considerar los constantes como una abstracción que surge de la forma en que se dan los sucedentes, se ajusta al concepto epistemológico según el cual es central poder identificar a los continuos en medio de la diversidad (Simons 2000b, p. 429). Podemos preguntarnos entonces qué es realmente primero: la manifestación de los constantes en medio del cambio o la continua transformación de los sucedentes, que eventualmente presentan ciertas regularidades.

Esto nos lleva a una nueva comparación entre Simons e Ingarden. Por una parte, en principio, el enfoque de Simons parece tener la ventaja de situar a los constantes directamente en el tiempo, como fragmentos de sucedentes, que por esencia son temporales. En cambio Ingarden se ve obligado a admitir una dualidad, con un núcleo ligado a la permanencia y una serie de estados que conectan este núcleo con los procesos en los que participa, permitiendo así situarlo en el tiempo. Pero la ventaja del esquema de Simons no es tan clara a este respecto. Si los constantes están determinados por la repetición de procesos "cerrados" dentro de sus límites, es igualmente necesario considerar las relaciones de dichos procesos con el conjunto global donde estos se dan. De no ser así, se dejaría "fuera del tiempo" a un conjunto particular de sucedentes, porque no sería posible situarlos en un tiempo " $\mathrm{t}$ " determinado. $\mathrm{Si}$, como indica el sentido común, lo que nos interesa es situar un constante en el tiempo, por ejemplo, "Juan estaba tal día en casa", entonces requerimos atender a un sucedente común a Juan y a otros distintos de Juan, y que se relacione con todos ellos, como por ejemplo, la marcha del reloj oficial del país.

\section{Esbozo de un nuevo análisis del tiempo}

La descripción de los procesos desarrollada por Ingarden parece al día de hoy muy natural, y podría incluso no demandar la existencia de objetos que perduran para su comprensión, a la inversa de lo que sostiene este autor. Esta sería la posición de Simons y de otros muchos contemporáneos. Desde un punto de vista metodológico, y evitando prolongar el debate acerca de la primacía ontológica de unos o de otros, mi propuesta consiste en explorar una posición intermedia, considerando los procesos y los objetos que perduran como dos aspectos complementarios de todo aquello que nos va ocurriendo como seres que se equilibran entre la permanencia y el cambio.

Atendiendo a los seres vivos y a la persona humana en particular, Ingarden describe con acuciosidad esta continua tensión entre lo permanente y lo transitorio. El largo análisis que dedica a establecer de qué forma los seres vivos pueden ser considerados como objetos que perduran en el tiempo, y la serie de importantes 
distinciones que va incorporando en su investigación, es, en mi opinión, una clara indicación de que también hay muchos elementos que nos podrían llevar a considerarlos como procesos complejos. El hecho de que el cambio sea consubstancial a los seres vivos lleva a Ingarden a postular un núcleo esencial que perdura y que se manifiesta en una identidad dinámica, pero cuya naturaleza habría que explorar, más allá de su pura cualidad de permanecer. Porque si el desarrollo del hombre se limita al modo y grado en que se manifiesta su núcleo esencial, permanente desde el inicio hasta el término de su existencia, se abren una serie de interrogantes, especialmente el problema del ámbito posible para la libertad humana.

Por otra parte, desde un punto de vista más general, parece no haber en el mundo real objetos que perduran absolutamente, pues hasta el Universo está en continua evolución, ni procesos "puros", porque un proceso requiere al menos una base referencial que no cambie mientras éste se encuentre en curso. Esto refuerza, entonces, la propuesta de no clasificar en forma estricta a los objetos en una de estas dos categorías, y considerarlas más bien al modo de límites, que nos permiten ordenar la multiplicidad que caracteriza lo que nos es presente cada vez, donde hay grados de permanencia y grados de transitoriedad. Considerando esta situación en que se dan la estabilidad y el cambio como un punto de inicio, es entonces necesario descubrir cómo es posible que lleguemos a concebir las ideas de objetos que permanecen y de procesos. Porque tal como Ingarden lo indica, la "condición de la posibilidad" de los procesos depende, al menos parcialmente, de los individuos (Ingarden 1964, p. 148). Y, por mi parte, sostengo que lo mismo podríamos agregar de los objetos que perduran.

Avanzando un paso más, considero necesario adoptar como punto de partida un análisis más detenido de la "fisura" en que la autoconciencia se abre a la duración abarcando por una parte lo ya pasado, retenido por la memoria, y por otra parte, lo por ocurrir, prefigurado por la imaginación. Porque si pensamos lo presente en la conciencia como una colección de instantes, a la manera de los eventos que define Ingarden, entonces la única forma de ordenarlos será indexarlos respecto de un tiempo absoluto, como cuando la física describe la posición de un objeto, $\mathrm{x}(\mathrm{t})$. Para cada t, hay una posición $\mathrm{x}$ determinada. Pero cabe al menos una objeción a este esquema, porque en la vida ordinaria debemos expresar t en alguna unidad específica, por ejemplo, en minutos, y un minuto, como es bien sabido, tiene sesenta segundos. Así que deberíamos medir en segundos, si queremos ser precisos. Pero a su vez un segundo consta, por ejemplo, de mil milisegundos, y así podríamos seguir. Así que cada t, en realidad es un rango, y no un punto, lo que en principio nos lleva a renunciar a la posibilidad de definir el tiempo como una serie de instantes "puros".

La otra opción es considerar la duración, donde podemos decir que tenemos conocimiento directo del antes y después de algo simultáneamente (aunque ello parece contradictorio a primera vista). Hay una multiplicidad de elementos que se nos van haciendo presentes, de tal manera que unos están ya dejando de ser, otros están siendo plenamente y otros están comenzando a ser, constituyendo un entrelazamiento de duraciones, y no un agregado de instantes. Sabemos de un conjunto múltiple de elementos, que distinguimos unos de otros en cuanto a su forma, y también en cuanto a su orden en que se van "haciendo presentes". Un ejemplo sencillo es una palabra: 
no necesitamos asociar cada letra a un tiempo para saber de su orden secuencial, sino que lo captamos de inmediato. Al leer o escuchar una palabra no hacemos: $\mathrm{H}\left(\mathrm{t}_{1}\right), \mathrm{o}\left(\mathrm{t}_{2}\right)$, $1\left(\mathrm{t}_{3}\right), \mathrm{a}\left(\mathrm{t}_{4}\right)=$ Hola. Sabemos que alguien dice "Hola" de un golpe, por así decirlo. Así pues, de acuerdo con nuestra experiencia parece más bien primar la duración, y no los instantes. Por ello, a mi juicio muy acertadamente, Ingarden indica que los procesos permiten entender más propiamente la naturaleza del tiempo, y no los eventos, que están vinculados conceptualmente con los instantes sin duración.

Ahora bien, ¿cómo distinguimos lo que cambia de lo que no lo hace, si todo lo que nos es presente no es sino procesos en desarrollo? Nada habría de permanente que sirva de referencia o de "soporte" del cambio, como objeta Ingarden. Ante este problema, considero que es necesario reformular la pregunta, porque la distinción no es entre lo que cambia y lo que no lo hace, sino entre lo que cambia más y lo que cambia menos (en la línea del precario equilibrio descrito por Ingarden para los seres vivos en relación con los procesos), o entre lo que permanece sin cambio significativo respecto de aquello que sí presenta modificaciones. En último término, debemos siempre atender a aquello que constituye nuestra identidad como personas autoconscientes, capaces de observar el cambio y la permanencia, desde la referencia implícita de nuestra propia estabilidad. Un análisis en esta dirección permitiría no solo esclarecer nuestro entendimiento del tiempo y del cambio, sino también el origen de nuestra identidad como personas autoconscientes y su relación con lo que se diferencia de ésta, a saber, el "mundo real" que tan relevante es para Ingarden. En cuanto a esto último, me parece importante mencionar, aunque sea solo de paso, que la idea de realidad implica una distinción respecto de aquello que no lo es. Por consiguiente, en este punto habría que atender al menos a la contrastación que realizamos en forma permanente entre lo proyectado por el ser consciente, pero ya en su calidad de pasado (como predicción de futuro ya cerrada, retenida por la memoria), con lo que efectivamente se está dando (como presente extendido, fisurado), que está en la base de toda interacción consciente.

\section{Referencias bibliográficas}

Husserl, E. (1993), Ideas relativas a una Fenomenología Pura y una Filosofía Fenomenológica. Traducción de J. Gaos. Madrid: Fondo de Cultura Económica. (2002), Lecciones de Fenomenología de la Conciencia Interna del Tiempo. Traducción de A. Serrano de Haro. Madrid: Editorial Trotta, S. A.

Ingarden, R. (1964), Time and Modes of Being. Traducción de H. R. Michejda. Springfield, Illinois: Charles C. Thomas.

Ingthorsson, R. D. (2009), “Can things endure in tenseless time?", Sats Nordic journal of philosophy 10: 79-99.

Kant, I. (1998), Crítica de la Razón Pura. Traducción de P. Ribas. Madrid: Grupo Santillana de Ediciones, S. A. 
Mitscherling, J. (2010), "Aristotelian Metaphysics and the Distinction between Consciousness and the Real World in Husserl and Ingarden", Polish Journal of Philosophy IV (2): 137-156.

(1997), Roman Ingarden's Ontology and Aesthetics. Ottawa: University of Ottawa Press.

Piwowarczyk, M. (2010), "Endurance and Temporality”, Polish Journal of Philosophy IV (2): 157-169.

Simons, P. (2000a), "Continuants and Occurrents, Part I", Proceedings of the Aristotelian Society, Supplementary Volumes 74: 59-75.

(2000b), "How to Exist at a Time When You Have No temporal Parts", The Monist 83 (3): 419-436.

Strózewski, W. (2010), "Roman Ingarden: Life and Philosophy", Polish Journal of Philosophy IV (2): 11-34.

Torretti, R. (2005), Manuel Kant: Estudio sobre los fundamentos de la filosofía crítica. Santiago de Chile: Ediciones Universidad Diego Portales.

Tymieniecka, A.-T. (1976), “Beyond Ingarden's Idealism/Realism Controversy with Husserl - The New Contextual Phase of Phenomenology", en T. ed., Analecta Husserliana IV (pp. 241-418). Dordrecht-Holland: D. Reidel Publishing Company. 